\title{
A Novel Fuzzy Algorithm to Introduce New Variables in the Drug Supply Decision-Making Process in Medicine
}

\author{
Jose M. Gonzalez-Cava $\mathbb{D}^{\mathbb{D}},{ }^{1}$ José Antonio Reboso, ${ }^{2}$ José Luis Casteleiro-Roca ${ }^{\mathbb{D}},{ }^{1,3}$ \\ José Luis Calvo-Rolle $\mathbb{D}^{3}{ }^{3}$ and Juan Albino Méndez Pérez $\left(^{1}{ }^{1}\right.$ \\ ${ }^{1}$ Department of Computer Science and System Engineering, Universidad de La Laguna (ULL), \\ San Cristóbal de La Laguna, 38200 Tenerife, Spain \\ ${ }^{2}$ Hospital Universitario de Canarias, San Cristóbal de La Laguna, Tenerife, Spain \\ ${ }^{3}$ Department of Industrial Engineering, Universidade da Coruña, Coruña, Spain \\ Correspondence should be addressed to Jose M. Gonzalez-Cava; jgonzalc@ull.edu.es
}

Received 30 November 2017; Revised 16 January 2018; Accepted 23 January 2018; Published 18 February 2018

Academic Editor: José Manuel Andújar

Copyright (C) 2018 Jose M. Gonzalez-Cava et al. This is an open access article distributed under the Creative Commons Attribution License, which permits unrestricted use, distribution, and reproduction in any medium, provided the original work is properly cited.

\begin{abstract}
One of the main challenges in medicine is to guarantee an appropriate drug supply according to the real needs of patients. Closedloop strategies have been widely used to develop automatic solutions based on feedback variables. However, when the variable of interest cannot be directly measured or there is a lack of knowledge behind the process, it turns into a difficult issue to solve. In this research, a novel algorithm to approach this problem is presented. The main objective of this study is to provide a new general algorithm capable of determining the influence of a certain clinical variable in the decision making process for drug supply and then defining an automatic system able to guide the process considering this information. Thus, this new technique will provide a way to validate a given physiological signal as a feedback variable for drug titration. In addition, the result of the algorithm in terms of fuzzy rules and membership functions will define a fuzzy-based decision system for the drug delivery process. The method proposed is based on a Fuzzy Inference System whose structure is obtained through a decision tree algorithm. A four-step methodology is then developed: data collection, preprocessing, Fuzzy Inference System generation, and the validation of results. To test this methodology, the analgesia control scenario was analysed. Specifically, the viability of the Analgesia Nociception Index (ANI) as a guiding variable for the analgesic process during surgical interventions was studied. Real data was obtained from fifteen patients undergoing cholecystectomy surgery.
\end{abstract}

\section{Introduction}

Artificial Intelligence (AI) plays an important role in science and engineering. This methodology is able to make decisions after a training process based on learning from a dataset obtained through expertise. One of the possible definitions of Artificial Intelligence refers to cognitive process and, specifically, to reasoning. Consequently, there is a natural relationship between Artificial Intelligence and decision-making [1]. Great progress has been made in different fields as industrial engineering $[2,3]$, tourist sector [4, 5], or energy field [6].

Specifically, in medicine, AI techniques have been applied with different aims. It includes the capability of learning automatically from data to control the health management systems, including an active guidance of clinicians in their treatment decisions. For clinical decision support, the key idea of the training process is extracting the expert knowledge from the information concerning medical records and the unstructured data including natural language [7]. E-health systems have become popular as they automatically evaluate the situation of patients without involvement from a physician [8]. Decision-making process in hospital management for prioritization of risks and assessment of failures has been also approached $[9,10]$. Moreover, AI has been used to automatic diagnosis and classification of illness [11, 12] and also for medical sensors fault detection [13]. Specifically, in medicine, the classifiers proposed to support a decisionmaking process must be suitable for being understood and 
evaluated from a clinician point of view [14]. Fuzzy rule-based systems have been widely used in medicine as they consist of simple linguistic rules that relate concepts in a natural manner $[15,16]$.

One of the main challenges in medicine is related to personalising the drug dose according to the real needs of patients. In most cases, the information obtained from the variable of interest leads to an increment or decrement of the drug infusion according to the medical criteria. AI has been also applied to automate the administration of drugs in medicine [17-20]. Important results have been reached in vasopressor administration [21,22] or control of anaesthesia [23-26]. The key idea of these systems is a closed-loop scheme in which a controller decides the drug dose comparing the information of the measured variable to the proposed target. To design an appropriate control structure, it is necessary to deal with a well-known process. As a result, it is difficult to automate those processes in which the control variable cannot be easily measured or the relationship between the drug infusion and the effects on patient is not well established.

Nowadays, different clinical monitors are being developed in order to propose new variables to improve the decision-making process in medicine. However, trying to establish a strict criterion to correlate the new information with the physician's action based on traditional clinical variables is not an easy task. The main objective of this research was defining a novel general methodology capable of studying the feasibility of a new clinical variable (controlled variable) to guide the drug delivery process and then designing automatically a fuzzy-based decision system taking this new information into account. Firstly, the accuracy of the new monitor to guide the drug infusion should be analysed. Then, the relationship between the new measurement and the physician criteria based on their expertise can be automatically proposed. The resulting Fuzzy Inference System based on a set of rules and membership functions makes it possible to obtain an easily interpretable drug delivery protocol for the clinician. A four-step methodology was proposed.

(i) Data collection for training process

(ii) Preprocessing and analysis of data

(iii) Designing the Fuzzy Inference System through a decision tree algorithm

\section{(iv) Validation of the results obtained}

There are different possible scenarios in which our algorithm could be applied. Specifically, to test the methodology above, the analgesia drug delivery process was analysed. Although different commercial monitors have been proposed, the main problem for the analgesia control is the absence of a reliable monitor to measure pain in patients undergoing surgery $[27,28]$. In this research, the suitability of the Analgesia Nociception Index (ANI) to guide the analgesic process under surgery was analysed. Training data were obtained from 15 patients undergoing cholecystectomy surgery. The paper is organised in the following way. The next section presents a detailed problem description as a starting point for this research. Section 3 provides a detailed explanation of the methodology proposed in this paper.
Section 4 presents the application of the methodology to the analgesia control field. Section 5 presents the results of the method. Section 6 includes the discussion of the results. Finally, in Section 7 we conclude the paper.

\section{Problem Description}

Delivering an appropriate amount of drug according to the real state of patient is such a hard task in medicine. Generally, physician evaluates the current state of the patient by means of specific monitors or using different clinical signs. Then, they decide whether it is necessary to change the drug dose. It is important to use the appropriate concentrations of medications to optimize clinical outcomes in patients in various clinical situations $[29,30]$. However, finding the variable that can be directly related to the effect of drug is not a trivial problem.

As a matter of fact, a new trend has been based on the proposal and development of new variables, techniques, and monitors capable of offering new information that may be included in the decision-making process. However, are these new measures directly related to the process involved? How could we define a new drug supply protocol in order to include this new information? These are the questions that this research aims to answer. As a result, the main objectives of this paper are as follows:

(1) Determining not only if the new controlled variable is able to guide the drug supply process but also which information should be specifically considered

(2) Defining a rule-based decision system in order to guide the supply process taking the new information into account.

Actually, there are a lot of fields in which the development of this algorithm would result in a success: glucose monitoring [31, 32], anaesthesia [33], or therapeutic drug monitoring $[34,35]$. In this research, we have focused on the control of analgesia. Optimizing the dose of opioid may limit the risk of overdosing and the risk of postoperative hyperalgesia and may reduce the time of recovery after surgical procedure [36]. However, the evaluation of analgesia and, therefore, the nociception-antinociception balance during surgery is a challenge to address due to the absence of an objective measure for monitoring analgesia. Traditional methods for supplying opioids use nonspecific and nonsensitive methods based on simple changes in vital signs such as movement, tachycardia, or lacrimation [28]. Recently, different monitors have been developed for measuring analgesia during clinical interventions proposing different information as nociception measures: heart rate information [37], electromyogram [38], electroencephalogram [39], or electrical skin conductance [40]. Nevertheless, the reliability of these monitors has not been deeply studied in clinical practice to assert that there exists a variable directly related to analgesia $[27,41]$.

Analgesia Nociception Index is a measure based on heart rate variability (HRV) analysis. HRV has been shown in several studies to measure Autonomic Nervous System tone, strongly influenced by anaesthetic drugs [42]. ANI has been 
employed in several research in order to validate it as a device capable of measuring the nociception balance [43-46]. ANI seems more sensitive than other traditional measures based on hemodynamic response of patient under propofol interventions to moderate nociceptive stimuli [47]. Using the ANI monitor as a guidance variable for analgesic titration may reduce the time recovery after the intervention, as well as the consumption of the analgesic agent $[48,49]$. Moreover, ANI may enable consistent reflection of stimulation during propofol-remifentanil anaesthesia, improving detection of a possible inadequate nociception/antinociception balance [50].

For conscious patient, the sympathetic-parasympathetic balance is affected by psychological stress. Using ANI in this case does not exclusively detect nociception but may be modified by stress and emotion [51, 52]. In general, further research is needed to evaluate whether ANI is a tool able to provide beneficial effects to the patients during anaesthesia. Traditional studies tend to compare ANI information with postoperative patient's painful experience to validate the ANI monitor. Visual Analogue Scale (VAS) is a standard measurement tool in pain research and clinical practice [53, 54]. It is supposed that changes in VAS score represent a relative change in magnitude of pain sensation. However, trying to establish a correlation between the ANI index during surgery and the postoperative evaluation of pain through VAN is influenced by pain subjective experience of patients [55]. Another trend is studying the variation of the monitor's measure through the application of painful stimuli to the patient [56], a clinical practice that may damage the patient's health. As a result, more research is needed to find a feasible method to test the validity of the different alternatives proposed. Although no analytical relationship has been proposed between drug infusion and ANI index, very promising results have been reached when using ANI as guidance variable in analgesia $[57,58]$. The algorithm developed in this research will be applied to propose a new solution to the analgesia problem from the AI point of view.

In light of the above, applying the novel algorithm proposed in this research to the control of analgesia will result in

(i) Determining whether the information displayed by the Analgesia Nociception Index is suitable to guide the analgesic process

(ii) Defining a Fuzzy Inference System considering the information displayed by the ANI capable of predicting the actions of the clinician.

\section{Methods}

In the present study, decision trees as well as fuzzy logic techniques were used. The basis of these algorithms is introduced in the following subsections. The main idea was using the information of a decision tree to design a Fuzzy Inference System (FIS). This structure will be capable of predicting the expert's decisions after a training step based on real data when a new monitor is involved in a drug supply process. Although more recent machine learning techniques have emerged with the purpose of the automation of data analysis, fuzzy logic has been chosen in this study for several reasons. Firstly, most of medical decisions when changing the drug dose cannot be based on crisp values or strict predefined criteria. Thus, fuzzy values due to the presence of ambiguous concepts in the decision making process such us interpatient variability or the existence of a lack of knowledge behind the process are required. That is why using membership functions in order to define the different categories for the decision variables seems to be the most appropriate option for the decisionmaking process. On the other hand, fuzzy logic is a wellknown method able to relate easily the heuristic knowledge to a set of rules in a natural manner. What is more, no complex mathematical modelling is needed as it is based on a linguistic characterisation of the quality of the controlled process. Obtaining a Fuzzy Inference System automatically through the algorithm we propose will result not only in the development of an automatic system for the drug supply trained with real data, but also in the definition of the basis of the process by means of a set of rules easily interpretable for clinicians.

The general scheme of the method proposed to design the decision-making system is shown in Figure 1.

One of the key steps in this methodology is the data collection. Data displayed by the new monitor must be recorded in parallel to the traditional drug supply process. It is important to note that the new monitor involved in this process should compute a numerical index in order to be able to apply the novel algorithm. To avoid conditioning the expert decision, the new information displayed must be hidden. Then, a preprocessing step is performed. Several proposals of the input data including different characteristics of the new measure must be considered. A decision tree algorithm is trained using the data recorded. The rules obtained will be the base to design the Fuzzy Inference System to predict the dose changes. On the one hand, it is possible to study the performance of the algorithm when trying to relate the new measure to the physician's actions. As a result, a first approach of the reliability of the new measure to guide the drug supply can be reached. Moreover, it is possible to determine which input proposal fits better to the decision-making process. On the other hand, the resulting Fuzzy Inference System consists of a set of rules whose interpretability improve the "userfriendliness" of the drug delivery protocol.

3.1. Decision Tree. Decision tree is a supervised machine learning algorithm able to build a model that makes predictions based on a known set of input data and known responses (output). The goal is to assign a class (categorical variable) from a finite set of classes to an observation. The decision tree consists of tests nodes linked to two or more subtrees and leafs or decision nodes labelled with a class which means the decision [59]. An instance is classified by starting at the root node of the tree. If the node is a test, the process continues with one of the subtrees. On the other hand, when a leaf is reached, the instance is classified with the correspondent label. An attribute node has exactly as many branches as its number of different value classes. Different algorithms to induce decision trees have been proposed $[60,61]$. The 


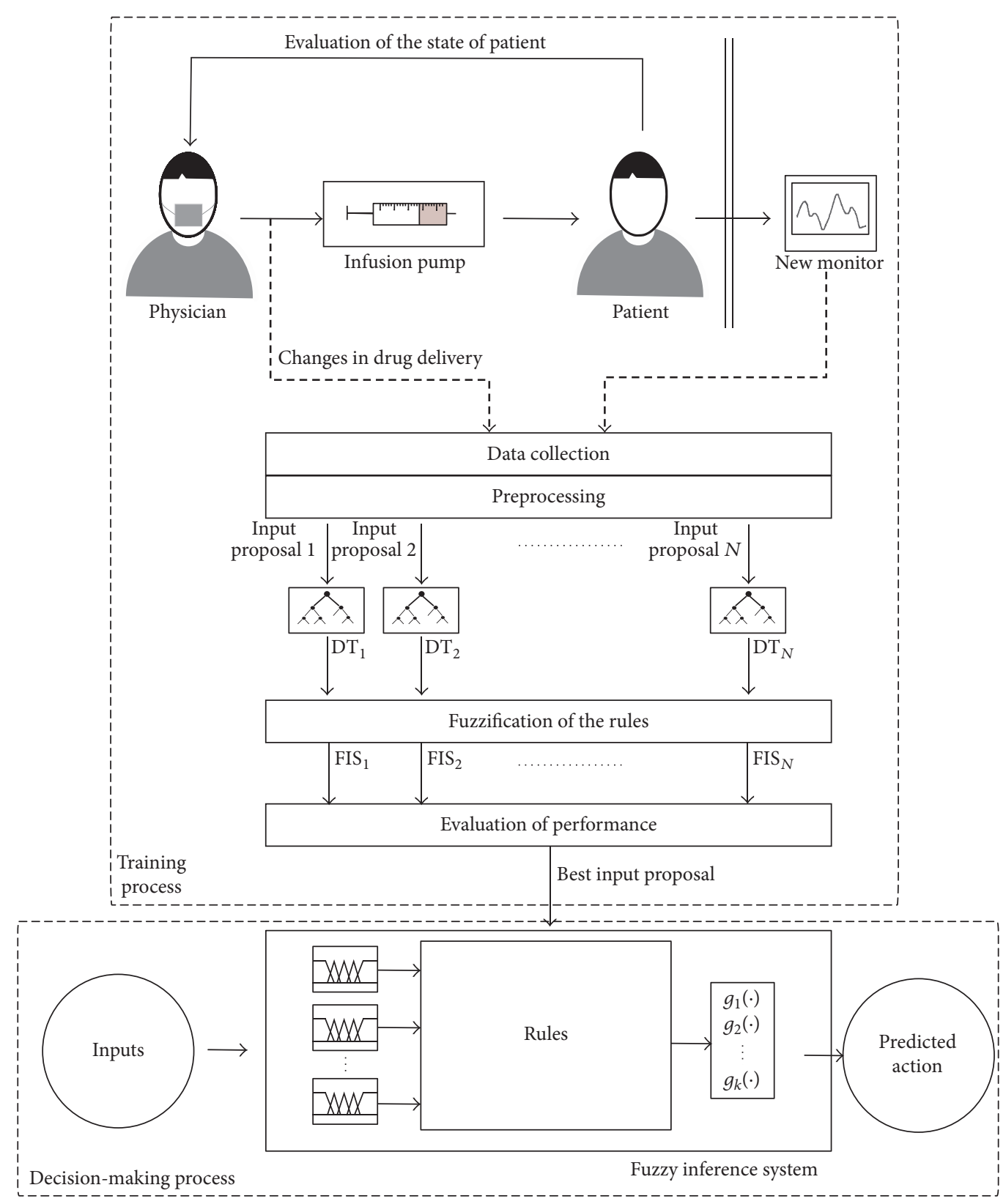

FIGURE 1: General scheme of the algorithm proposed in this study.

main idea relies on using statistical calculation of information gain from the attributes. As a result, attributes adding the most information about the decision are selected first in the decision tree construction.

For this research, a CART (Classification and Regression Trees) algorithm was proposed. This method introduced by Breiman et al. [62] is focused on minimising the relative sum of squared errors in the two partitions resulting from a split. Generally, a two-step process is developed: a preliminary induction of the model through a training set under the "divide and conquer" principle and a checking process of the accuracy from a testing set. The search for splits in CART is based on two main characteristics: the covariate to split on and splitting point within that covariate [63]. Firstly, trees are grown to a maximal size stopping when no further splits are possible due to the lack of data [64]. Gini, similar to entropy criterion, is used as the splitting rule for classification. For a two-decision target the Gini measure of impurity of a node $t$ is given by the expression below.

$$
G(t)=1-p(t)^{2}-(1-p(t))^{2},
$$

where $p(t)$ is the relative frequency of one of the label in the node. Then, the tree is pruned back to the root based strictly on the training data according to a cost-complexity measure defined as

$$
R a(T)=R(T)+a|T|,
$$




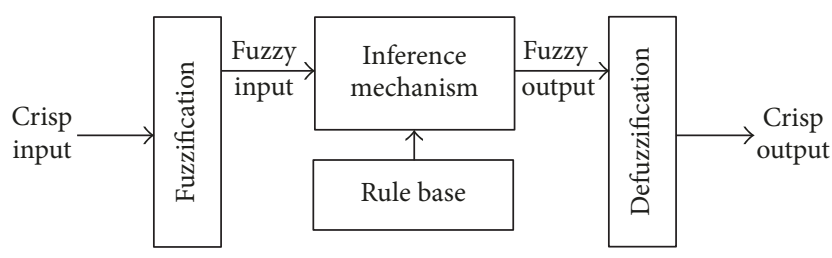

FIGURE 2: General structure of a Fuzzy Inference System.

where $R(T)$ is the training sample cost of the tree, $|T|$ is the number of terminal nodes, and $a$ is a penalty imposed on each node increasing from 0 to a value sufficient to prune away all splits. As a consequence, the next split to be pruned is the one that decreases the total performance of the tree.

3.2. Fuzzy Inference System. Fuzzy Inference System (FIS) is a fuzzy logic based structure capable of making decisions in real time taking human expert knowledge into account. The main idea is based on mapping the inputs and the outputs through a set of predefined rules that involves the heuristic knowledge. According to fuzzy sets theory, each variable (input or output) is defined through a linguistic variable $\tilde{u}_{i}$ whose value can be described through linguistic values $\widetilde{A}_{i}^{j}$ belonging to a universe of discourse $U_{i}$. Unlike crisp values, the values of the universe of discourse "belong to" a linguistic value in a certain degree $[0,1]$ described by a membership function $\mu\left(u_{i}\right)$.

$$
\mu_{A_{i}^{j}}\left(u_{i}\right)=X \longrightarrow[0,1] .
$$

A value near 1 indicates that the value is almost fully in the set. A fuzzification process is necessary to turn crisp values to a fuzzy value. The singleton fuzzification is the most commonly used method. Then, the mapping of the inputs to the output is characterised by if-then rules. An inference step is needed to obtain conclusions from inputs and rule base. In this study, a Takagi-Sugeno inference system was developed.

$$
\begin{aligned}
\text { IF } & u_{1} \text { is } A^{1}{ }_{i}, u_{2} \text { is } A^{2}{ }_{i}, \ldots, u_{n} \text { is } A^{n}{ }_{i}, \\
\text { THEN } & b_{i}=g_{i}(\cdot),
\end{aligned}
$$

where "." represents the argument of $g_{i}$ function. As a result, the consequence of a Takagi-Sugeno inference is a function that may include the input terms $u_{i}$. Finally, a defuzzification method is needed to obtain a crisp value of the output:

$$
y=\frac{\sum_{i=1}^{R} b_{i} \mu_{i}}{\sum_{i=1}^{R} \mu_{i}} .
$$

The general structure of a Fuzzy Inference System is shown in Figure 2.

3.3. Fuzzification of the Decision Tree Rules. Generally, when a decision must be made in medicine, there is not a predefined universal criterion. It is mainly due to the different inter- and intravariability characteristics that the process involves. As a result, it does not make any sense to consider a crisp value as a strict limit to make a decision. That is one of the main reasons why a FIS was introduced in this research. Furthermore, fuzzy logic is based on "categories" or membership functions easier to interpret for clinicians as it groups information with similar characteristics for the decision-making process.

One of the key steps when designing a FIS is related to the definition of the membership functions and the rule base. It is especially difficult when there is not a deep heuristic knowledge behind the process. To avoid this problem, a decision tree technique is proposed to obtain it automatically from real data. The limits of the membership functions will be defined through the conditions in the test nodes and the rules will inherit from it. However, while the limits of the decision tree are crisp values based on training data, fuzzy values are required for Fuzzy Inference Systems. In addition, when the number of training and testing data is limited, it can turn into a harder problem. In order to generalise our model and to take advantage of the fuzzy techniques, triangular and trapezoidal membership functions were used for intermedia and edge partitions of the universe of discourse, respectively. Moreover, we proposed to increase the limits of each membership function in $10 \%$ to get an overlap and avoid problems related to the limitation in the amount of data in the training step. The new limits for each membership function are calculated as shown below:

New lower limit $=$ lower limit

$$
-\frac{\text { upper limit }- \text { lower limit }}{2}
$$

$\cdot 0.1$

New upper limit $=$ upper limit

$$
\begin{aligned}
& +\frac{\text { upper limit }- \text { lower limit }}{2} \\
& \cdot 0.1 \text {. }
\end{aligned}
$$

The 2-step process for the fuzzification of the inputs is described in Figure 3.

Finally, the number of output functions matches the number of actions that the physician can handle. A constant function will be proposed for each action. For a general situation in which two decisions can be made (decreasing or increasing drug), a constant value of 0 and 100 could be associated with each action, respectively. In this case, the Fuzzy Inference System will calculate a number within the 0-100 range that could be considered as a percentage of action. A value of 50 could be regarded as the limit between both decisions.

3.4. Evaluation of the FIS Decision Maker. In order to evaluate the performance of the method proposed and the capability of the resulting Fuzzy Inference System to predict the decision-making process, a $k$-fold cross-validation must be performed [65]. The original sample is randomly divided into $k$ equal sized subsamples. A single subsample is considered as the validation data for testing the Fuzzy Inference System, while the remaining $k-1$ subsamples are used as 
1st step: application of decision tree

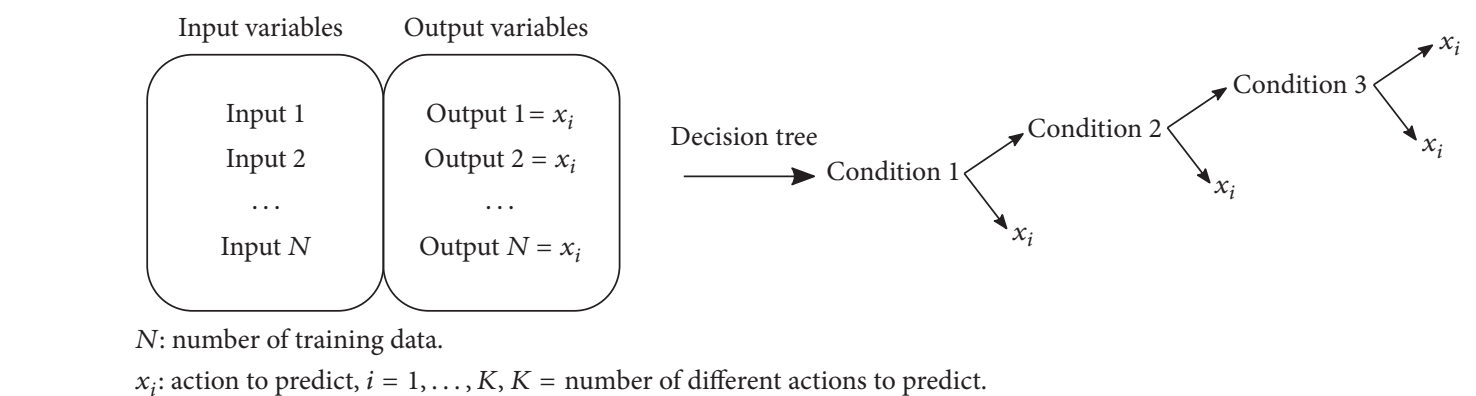

$x_{i}$ : action to predict, $i=1, \ldots, K, K=$ number of different actions to predict.

2nd step: fuzzification of the decision tree
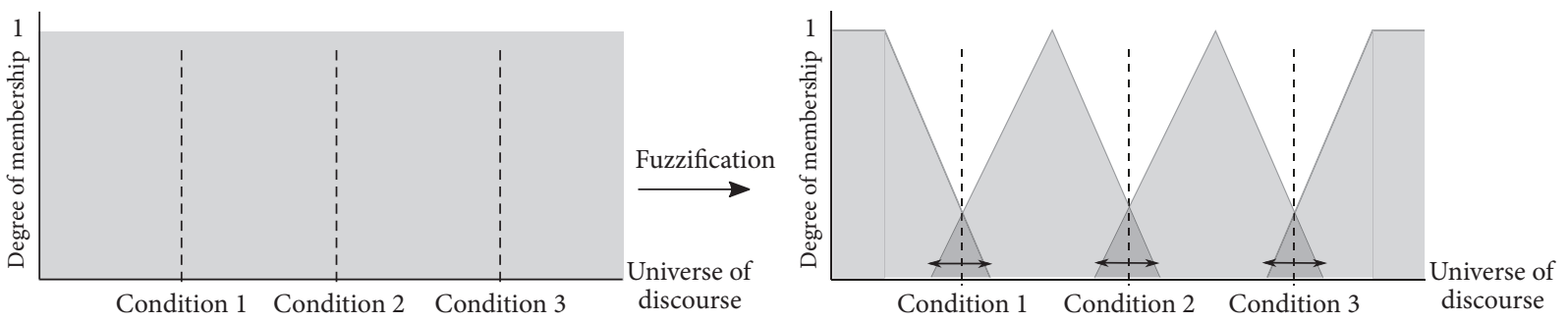

FIGURE 3: Graphic description of the Fuzzy Inference System definition process proposed in this research.

training data. The process is repeated $k$ times varying the validation data and the results are averaged to obtain a single estimation. Different measures are calculated to study the performance of the classification [66]. The accuracy indicates the percentage of the dataset that are correctly classified by the proposed classifier. The sensitivity and specificity calculate the proportion of positive and negative records that are correctly classified, respectively. Precision refers to the fraction of relevant instances among the retrieved instances while recall is the fraction of relevant instances that have been retrieved over total relevant instances. The mathematical expressions to calculate the different measures are shown below.

$$
\begin{aligned}
\text { Accuracy } & =\frac{\mathrm{TP}+\mathrm{TN}}{\mathrm{TP}+\mathrm{TN}+\mathrm{FN}+\mathrm{TN}} \cdot 100(\%) \\
\text { Sensitivity } & =\frac{\mathrm{TP}}{\mathrm{TP}+\mathrm{FN}} \\
\text { Specificity } & =\frac{\mathrm{TN}}{\mathrm{TN}+\mathrm{FP}} \\
\text { Precision } & =\frac{\mathrm{TP}}{\mathrm{TP}+\mathrm{FP}} \\
\text { Recall } & =\frac{\mathrm{TP}}{\mathrm{TP}+\mathrm{FN}} .
\end{aligned}
$$

Given two classes, TP (true positives) refer to the positive records that have been correctly classified by the FIS, while $\mathrm{TN}$ (true negatives) are the negative records that have been correctly labelled by the classifier. On the other hand, FP (false positives) are the negative records that have been incorrectly labelled, while FN (false negatives) refers to the positive records incorrectly classified by the FIS. The
TABLE 1: Confusion matrix for positive and negatives records.

\begin{tabular}{ccc}
\hline & & Predicted \\
& Positive & Negative \\
\hline Observed & & \\
Positive & TP & FN \\
Negative & FP & TN \\
\hline
\end{tabular}

confusion matrix to define the different measures is shown in Table 1.

Different input proposals based on different information from the new monitor are considered in the algorithm. In order to choose the best input proposal for the decision making process, the evaluation of the input variables resulting in the highest accuracy, sensitivity, specificity, precision, and recall will be considered for the final FIS. To determine whether the information provided by a new monitor is relevant for a specific decision-making process, the measures of performance should be compared with the results obtained in similar previous research based on traditional decision methods or, if it was not possible, being evaluated by an expert.

\section{Analgesia Assessment Application}

This study has been approved by the Ethics Committee for the Clinical Research of the Hospital Universitario de Canarias (2014-97 (760954923-54923-4-14)). After obtaining written informed consent from patient, fifteen patients undergoing cholecystectomy surgery were enrolled in this study. A total intravenous anaesthesia (TIVA) with propofol (hypnotic) and remifentanil (analgesic) was performed for induction and maintenance of general anaesthesia. A Bispectral Index (BIS) 


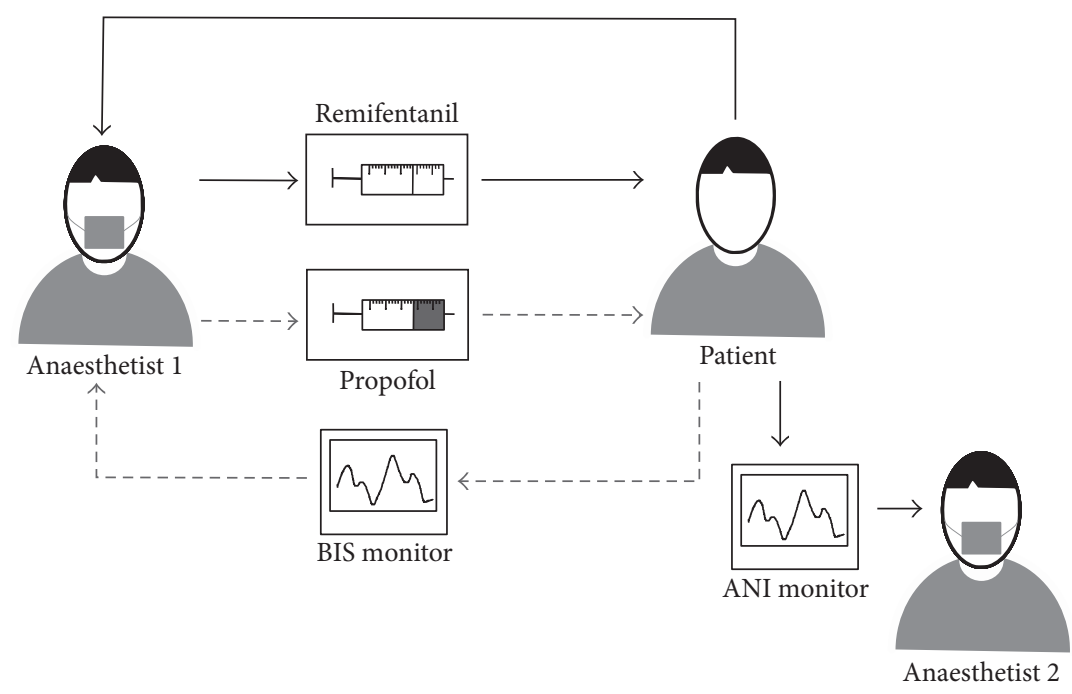

- Analgesia control
- - - Hypnosis control

FIGURE 4: Scheme of the data collection process during the surgical interventions.

monitor (Aspect Medical Systems Inc., Newton, MA, USA) was used as guidance variable for propofol titration. The propofol dose was changed during the surgery to maintain BIS values between 40 and 60 , with a target of 50 . The target dose of remifentanil was adjusted at the discretion of the anaesthesiologist, according to clinical practice parameters, anticipation to surgical stimuli, reactivity, or hemodynamic events. The dose of remifentanil was adjusted in steps of $0.05-0.1 \mathrm{mcg} \mathrm{kg}^{-1} \mathrm{~min}^{-1}$.

4.1. The Analgesia Nociception Index. The Analgesia Nociception Index (ANI), developed by Mdoloris Medical System, is a noninvasive system that displays a continuous index related to the Autonomic Nervous System (ANS) through the heart rate variability. ANI is supposed to be a monitoring system of the parasympathetic activity that displays information about the level of pain or stress in patients undergoing surgery. ANI index computation is based on a frequency domain analysis of the ECG signal. The main idea leads to studying the spectral content of RR waves series after a preprocessing step focusing on the high frequency range only influenced by the parasympathetic tone. Specific ECG electrodes are placed on the chest or back of patient to collect the heart rate variability. Every second two measures ranging from 0 to 100 are displayed: instant ANI and mean ANI. Instant ANI is directly related to the reactions of the patient to painful stimuli while mean ANI, computed after two minutes of averaging instant ANI, is related to the effects of analgesia on a patient. As a result, instant ANI may detect the actions of the surgeon and mean ANI could be useful for the titration of analgesia. Target values between 50 and 70 for mean ANI have been proposed to avoid unwanted hemodynamic events. Values under 50 increase the possibility of hypertension, hypotension, tachycardia, or bradycardia events.
4.2. Data Collection and Preprocessing. According to the process described in Methods, the data collection is the first step of the proposed methodology. In this case, two anaesthetists took part in each surgery. One of them was in charge of the drug supply, while the second one supervised the data recording process in a computer. A software in Matlab was developed in order to collect the data automatically. The scheme of the process is shown in Figure 4. Information displayed by the ANI monitor was hidden in order to avoid conditioning the decisions of the first anaesthetist. Instant ANI, mean ANI, and remifentanil dose changes $\left(\mathrm{mcg} \mathrm{kg}^{-1} \mathrm{~min}^{-1}\right)$ were recorded every five seconds. Predefined surgical stimuli were also registered: nasogastric tube, laryngoscopy, incision, trocars, and the creation of pneumoperitoneum.

A postoperative offline study was made to try to correlate the rate changes of remifentanil with the ANI values. Before the analysis, a data preprocessing was necessary. On the one hand, zero-index value because of poor signal or external disturbances was corrected through a linear interpolation algorithm. On the other hand, only changes of remifentanil due to the analgesic state of patients were considered in this study. Changes of remifentanil rate during surgery were based on two criteria: the anticipation to predefined painful surgical stimuli and the analgesic state of patient. As far as ANI is not able to predict the changes based on the anticipation to surgical stimuli, these values were not considered in this study.

4.3. Decision Variables. The accuracy of the algorithm prediction will be directly related to the combination of the inputoutput variables proposed. As a result, different information obtained through the ANI index was tried to establish a relationship with the action of the anaesthetist. Firstly, a categorical variable was considered for the output. Consequently, 
TABLE 2: Description of the input variables proposed for the study.

\begin{tabular}{ll}
\hline Variables & Description \\
$\mathrm{ANI}_{i 20}$ & Instant ANI of last 20 samples $(100 \mathrm{~s})$ \\
$\mathrm{ANI}_{m}$ and $\mathrm{ANI}_{i 5}$ & Last mean ANI and last 5 samples of instant ANI $(25 \mathrm{~s})$ \\
$\mathrm{ANI}_{m 5}$ and $\mathrm{ANI}_{i 10}$ & Mean ANI of last 5 samples $(25 \mathrm{~s})$ and instant ANI of last 10 samples $(50 \mathrm{~s})$ \\
$\mathrm{ANI}_{m 10}$ and $\mathrm{ANI}_{i 20}$ & Mean ANI of last 10 samples $(50 \mathrm{~s})$ and instant ANI of last 20 samples $(100 \mathrm{~s})$ \\
$\mathrm{ANI}_{m 10}$ and $\mathrm{ANI}_{i 30}$ & Mean ANI of last 10 samples $(50 \mathrm{~s})$ and instant ANI of last 30 samples (150 s) \\
Average $\mathrm{ANI}_{m 5}$ and $\mathrm{ANI}_{i 5}$ & Average of last 5 samples of Mean ANI $(25 \mathrm{~s})$ last 5 samples of instant ANI (25 s) \\
Average $\mathrm{ANI}_{m 5}$ and $\mathrm{ANI}_{i 20}$ & Average of last 5 samples of Mean ANI $(25 \mathrm{~s})$ last 20 samples of instant ANI (100 s) \\
Average $\mathrm{ANI}_{m 5}$ and $\Delta \mathrm{ANI}_{i 5}$ & Average of last 5 samples of Mean ANI $(25 \mathrm{~s})$ the increment of last 5 samples of instant ANI $(25 \mathrm{~s})$ \\
Average $\mathrm{ANI}_{m 5}$ and $\Delta \mathrm{ANI}_{i 20}$ & Average of last 5 samples of Mean ANI $(25 \mathrm{~s})$ the increment of last 20 samples of instant ANI (100 s)
\end{tabular}

"increasing drug" or "decreasing drug" labels were defined as it fully considers the anaesthetist's actions. Moreover, nonquantitative values of changes in remifentanil dose were analysed as the rate changes were limited by the clinical protocol (steps of $0.05-0.1 \mathrm{mcg} \mathrm{kg}^{-1} \mathrm{~min}^{-1}$ ).

For the input, different variables computed from instant ANI and mean ANI were taken into account. In addition, the effects of considering different time intervals for both variables were also analysed. Finally, to study the evolution of ANI, the increment of instant ANI was computed through the slope of the regression line that best matched the last values for a time interval. The variables proposed and their description are shown in Table 2.

\section{Results}

The decision-making methodology proposed was applied to the analgesic drug supply scenario. Fifteen patients undergoing cholecystectomy surgery were enrolled in this study. An example of the data collected during the interventions is shown in Figure 5. A total of 91 increasing/decreasing events were registered during the 15 surgeries. After discarding the changes due to the anticipation to painful stimuli, 53 events were finally considered for this study ( 32 increasing versus 21 decreasing).

5.1. Evaluation of the Proposed Variables. The performance of the resulting Fuzzy Inference Systems for both "increasing drug" and "decreasing drug" actions considering the different variables proposed in Section 4.3 is shown in Tables 3 and 4. A 4-fold cross-validation was applied for each combination according to the total number of training data.

In light of the results of the cross-validation, accuracy was over $60 \%$ for all the inputs considered. Specifically, accuracy was over $70 \%$ in most of the combinations proposed. Regarding the analgesia scenario, it was highly important to note that a low value of analgesia in patients can lead to complications and prolonged rehabilitation as well as the development of chronic pain with reduction in quality of life $[67,68]$. Consequently, accurate increments of remifentanil dose were desirable. That was why sensitivity and recall measures in Table 3 were specifically considered to choose the appropriate FIS. In this sense, there were up to four inputoutput combinations that resulted in sensitivity and recall

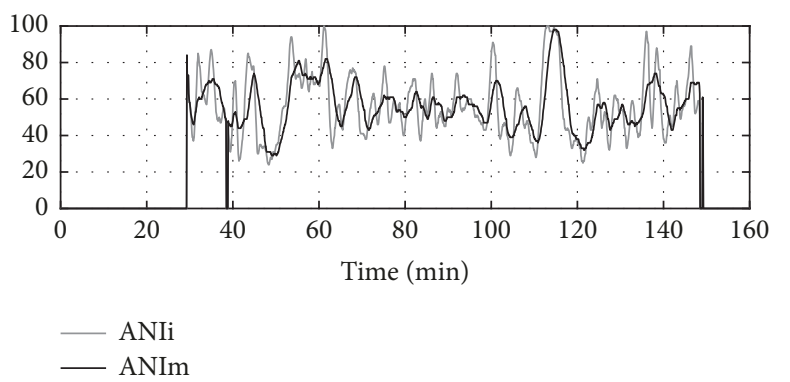

(a)

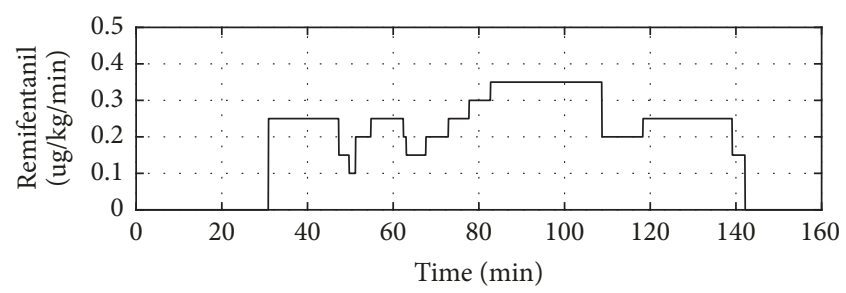

(b)

FIgURE 5: Example of the data collected for a patient undergoing cholecystectomy surgery. ANI registered (a) and remifentanil infusion rate (b). ANIi: instant ANI. ANIm: mean ANI.

values over 0.8 . Taking these four combinations into account as well as specificity and precision values, it was concluded that the best performance was reached when considering the average of the last 5 samples of mean ANI and the increment of the last 20 samples of instant ANI. Similar results were reached when analysing Table 4 , as this inputoutput proposal resulted not only in the highest sensitivityspecificity combination but also in the highest precision value.

As a result, the last 5 samples of mean ANI and the increment of the last 20 samples of instant ANI were chosen for the input of our decision-making system. Regarding the performance reached, it was possible to affirm that there existed a relationship between the actions of the anaesthesiologist during surgery and the values displayed by the Analgesia Nociception Index.

5.2. Structure of the Fuzzy Inference System. Taking into account the comparison of performances in Section 5.1, the 
TABLE 3: Comparison of performance for the different input combinations for when applying the proposed algorithm for increasing drug action.

\begin{tabular}{|c|c|c|c|c|c|}
\hline Input & $\begin{array}{c}\text { Accuracy } \\
(\%)\end{array}$ & Sensitivity & Specificity & Precision & Recall \\
\hline $\mathrm{ANI}_{i 20}$ & 71.57 & 0.83 & 0.65 & 0.80 & 0.83 \\
\hline $\mathrm{ANI}_{m}$ and $\mathrm{ANI}_{i 5}$ & 71.57 & 0.77 & 0.74 & 0.83 & 0.77 \\
\hline $\mathrm{ANI}_{m 5}$ and $\mathrm{ANI}_{i 10}$ & 66.07 & 0.69 & 0.71 & 0.80 & 0.69 \\
\hline $\mathrm{ANI}_{m 10}$ and $\mathrm{ANI}_{i 20}$ & 69.64 & 0.81 & 0.62 & 0.80 & 0.81 \\
\hline $\mathrm{ANI}_{m 10}$ and $\mathrm{ANI}_{i 30}$ & 71.57 & 0.76 & 0.72 & 0.83 & 0.76 \\
\hline Average $\mathrm{ANI}_{m 5}$ and $\mathrm{ANI}_{i 5}$ & 71.57 & 0.77 & 0.72 & 0.81 & 0.77 \\
\hline Average $\mathrm{ANI}_{m 5}$ and $\mathrm{ANI}_{i 20}$ & 71.57 & 0.83 & 0.64 & 0.80 & 0.83 \\
\hline Average $\mathrm{ANI}_{m 5}$ and $\Delta \mathrm{ANI}_{i 5}$ & 62.09 & 0.71 & 0.56 & 0.73 & 0.71 \\
\hline Average $\mathrm{ANI}_{m 5}$ and $\Delta \mathrm{ANI}_{i 20}$ & 75.41 & 0.82 & 0.71 & 0.80 & 0.82 \\
\hline
\end{tabular}

TABLE 4: Comparison of performance for the different input combinations for when applying the proposed algorithm for decreasing drug action.

\begin{tabular}{|c|c|c|c|c|c|}
\hline Input & $\begin{array}{c}\text { Accuracy } \\
(\%)\end{array}$ & Sensitivity & Specificity & Precision & Recall \\
\hline $\mathrm{ANI}_{i 20}$ & 71.57 & 0.65 & 0.83 & 0.5 & 0.65 \\
\hline $\mathrm{ANI}_{m}$ and $\mathrm{ANI}_{i 5}$ & 71.57 & 0.74 & 0.77 & 0.72 & 0.74 \\
\hline $\mathrm{ANI}_{m 5}$ and $\mathrm{ANI}_{i 10}$ & 66.07 & 0.71 & 0.69 & 0.62 & 0.71 \\
\hline $\mathrm{ANI}_{m 10}$ and $\mathrm{ANI}_{i 20}$ & 69.64 & 0.62 & 0.81 & 0.46 & 0.63 \\
\hline $\mathrm{ANI}_{m 10}$ and $\mathrm{ANI}_{i 30}$ & 71.57 & 0.72 & 0.76 & 060 & 0.72 \\
\hline Average $\mathrm{ANI}_{m 5}$ and $\mathrm{ANI}_{i 5}$ & 71.57 & 0.72 & 0.77 & 0.70 & 0.72 \\
\hline Average $\mathrm{ANI}_{m 5}$ and $\mathrm{ANI}_{i 20}$ & 71.57 & 0.64 & 0.83 & 0.51 & 0.64 \\
\hline Average $\mathrm{ANI}_{m 5}$ and $\Delta \mathrm{ANI}_{i 5}$ & 62.09 & 0.56 & 0.71 & 0.62 & 0.56 \\
\hline Average $\mathrm{ANI}_{m 5}$ and $\Delta \mathrm{ANI}_{i 20}$ & 75.41 & 0.71 & 0.82 & 0.74 & 0.71 \\
\hline
\end{tabular}

analysis and the result of the final FIS regarding the inputoutput proposal with the highest prediction score (the last 5 samples of mean ANI and the increment of the last 20 samples of instant ANI) are studied in this section. On the one hand, the decision tree obtained is shown in Figure 6. The value of the input variables proposed for the 53 registered events are shown in Figure 7.

Triangular as well as trapezoidal membership functions were used for both inputs. The number of the membership functions were defined by the total number of test nodes associated to each input. The limits, inherited from the test node conditions, were fuzzified according to the criteria in Section 3.3. The results are shown in Figure 8. A number of four and two membership functions were defined for the average $\mathrm{ANI}_{m 5}$ and $\Delta \mathrm{ANI}_{i 20}$ inputs, respectively.

Finally, the output functions of the FIS were defined. In this case, a two-decision system was needed: "increasing drug" and "decreasing drug" actions. Two constant output functions were proposed: " 0 " and " 100 " referred to the decreasing and increasing actions, respectively. As a result, the output of the FIS was a number within 0-100 range which could be regarded as a percentage of action. In this study, the results over 50 were considered as an "increasing drug" prediction while the values under 50 were considered as "decreasing drug" prediction. The fuzzy decision surface obtained and the comparison with the nonfuzzified decision tree surface are shown in Figure 9.

To evaluate the performance of the FIS, the decision system was evaluated through a receiver-operating characteristic (ROC) curve by plotting the sensitivity, or true positive rate as a function of the false-positive rate. The ROC curves for both increasing and decreasing predictions are shown in Figure 10. An Area Under the Curve (AUC) of 0.8557 was reached for the predictive model proposed.

Finally, the prediction of the Fuzzy Inference System developed for the 53 training data is shown in Figure 11.

\section{Discussion}

A new algorithm for the design of a computer-based decision system in medicine has been presented. Specifically, the application of the proposed methodology in this research has resulted in the development of a Fuzzy Inference System as a computer-assisted medical decision-making for the analgesia scenario. On the one hand, it was possible to determine that there existed a relationship between the Analgesia Nociception Index and the remifentanil supply during surgery. Particularly, the average of last five samples of mean ANI and the increment of last twenty samples of instant ANI reached an accuracy of $75.41 \%$. Moreover, sensitivity and recall values 


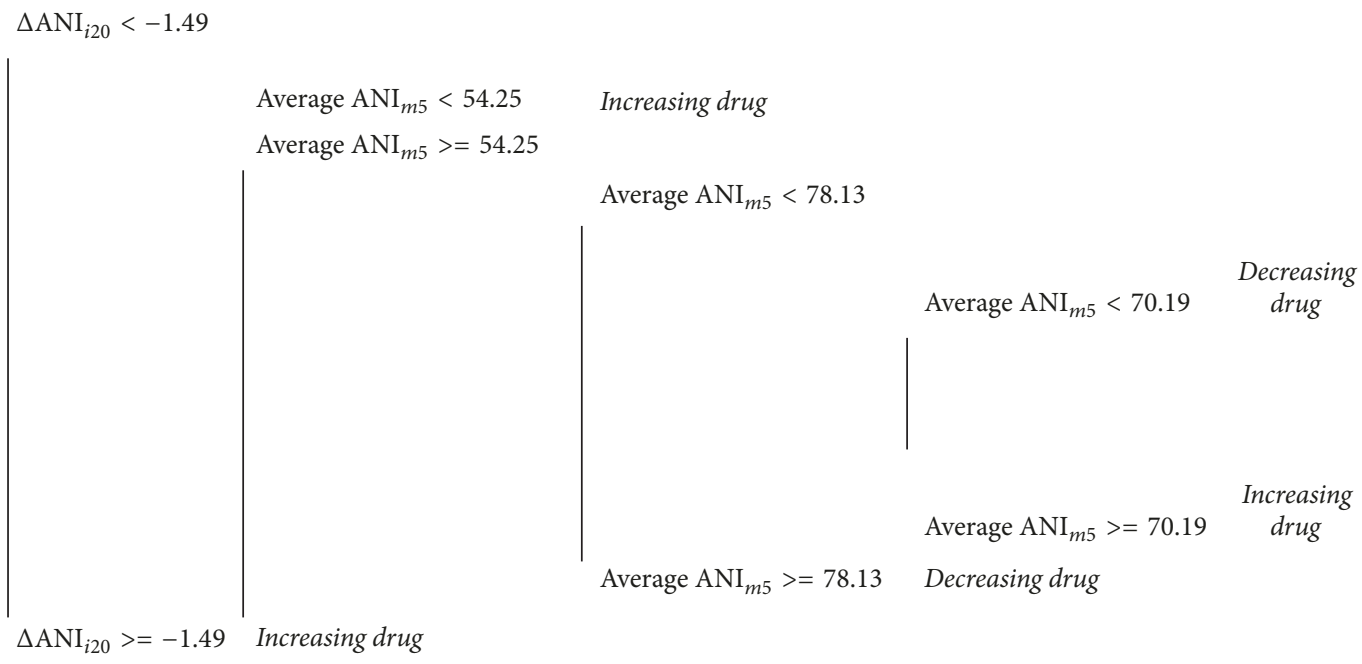

FIGURE 6: Decision tree obtained for the best input-output proposal (the last 5 samples of mean ANI and the increment of the last 20 samples of instant ANI) when applying the algorithm.

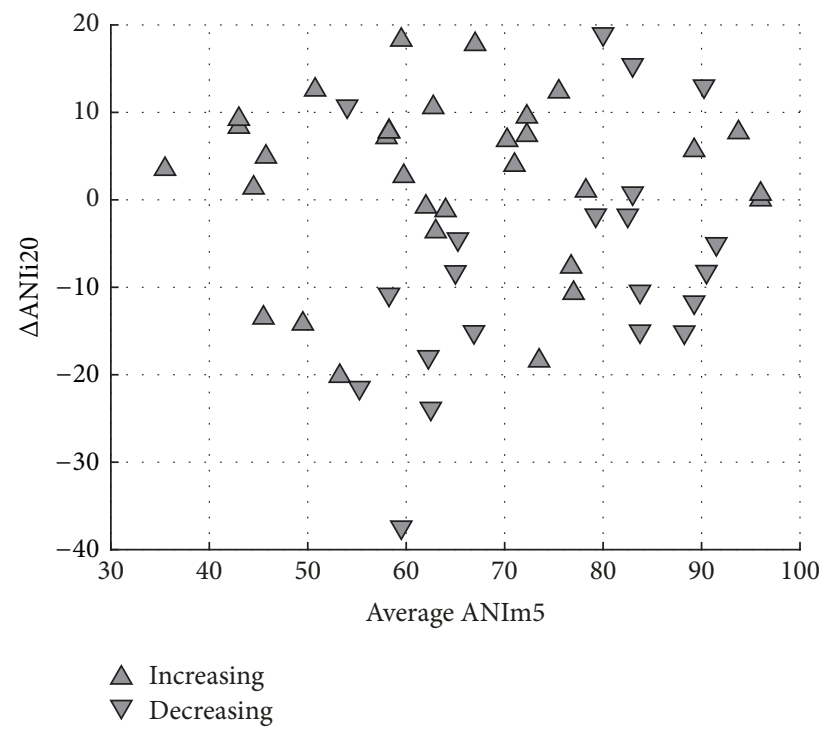

FIGURE 7: Value of average $\mathrm{ANI}_{m 5}$ and $\Delta \mathrm{ANI}_{i 20}$ computed for every remifentanil rate change (increasing drug or decreasing drug).

over 0.8 were reached when predicting the increments of remifentanil. Similar performance has been reached when applying different machine learning algorithms to optimize drug supply in medicine. Specifically, an accuracy ranging from $75 \%$ to $88 \%$ was obtained after a leave-one-out study when predicting the discrepancies between planned and delivered dose in proton therapy [69]. In [70], the overall predictive accuracy of the presented models for prediction of optimal cancer drug therapies was $80 \%$. In the analgesia field, the development of models to predict the postoperative pain treatment reached an accuracy of 65\% [71]. According to previous research, a clinically acceptable accuracy level is reached when applying our proposal. A two-input one-output FIS based on Takagi-Sugeno inference was developed. The resulting model reached an AUC of 0.8557 for both increasing

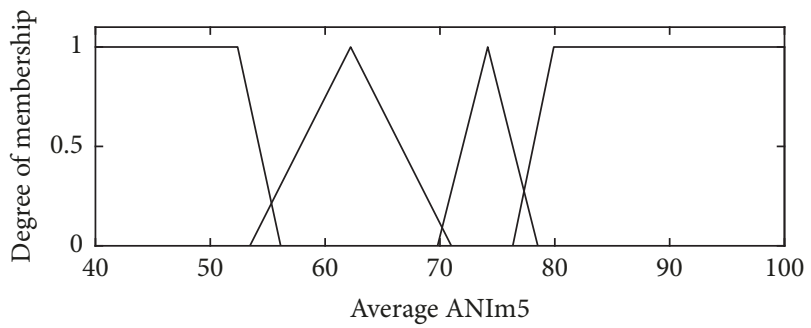

(a)

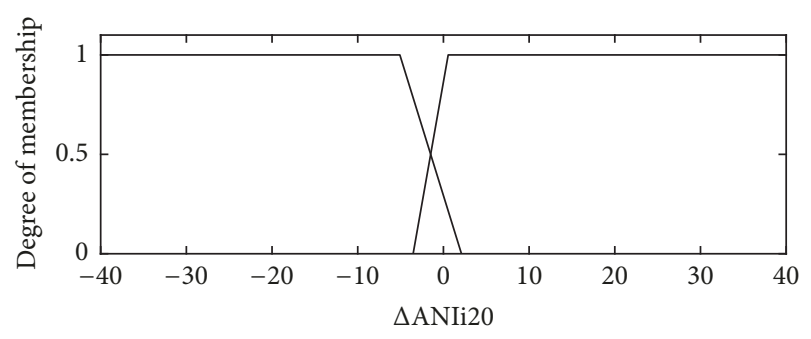

(b)

FIGURE 8: Input fuzzy partitions of the Fuzzy Inferences System. (a) Membership functions for average $\mathrm{ANI}_{m 5}$ input. (b) Membership functions for the $\Delta \mathrm{ANI}_{i 20}$ input.

and decreasing drug actions. As a result, the performance of the model to predict the actions of the anaesthesiologist may be classified as good [72].

From the Artificial Intelligence point of view, different approaches have been also proposed to fuzzy rule extraction from numerical data for classification [73-75]. However, the main novelty of this research is the definition not only of an automatic algorithm but also of the whole process in order to evaluate the reliability of a monitor involved in a decisionmaking system. As far as we know, this is the first study that tries to establish a correlation between the information displayed by a monitor and the expertise-based decisionmaking process during surgical interventions. Consequently, 


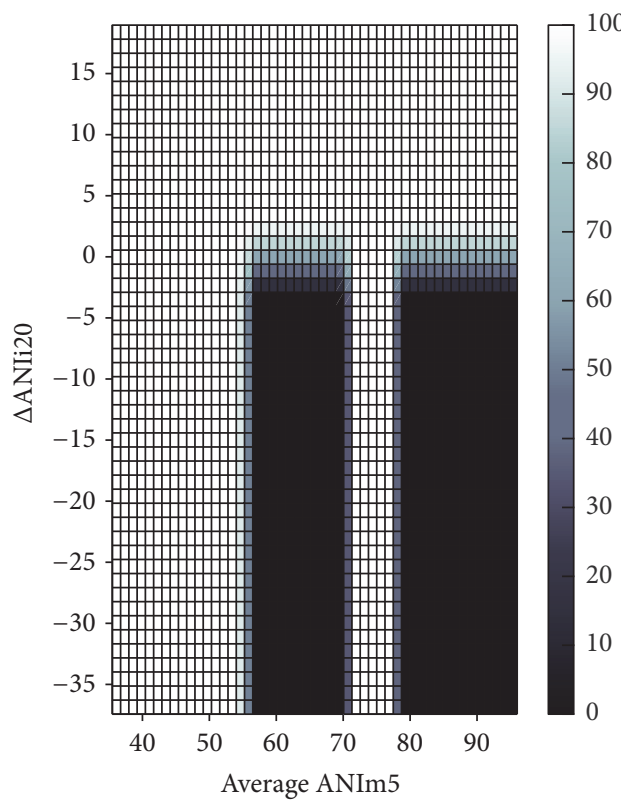

(a)

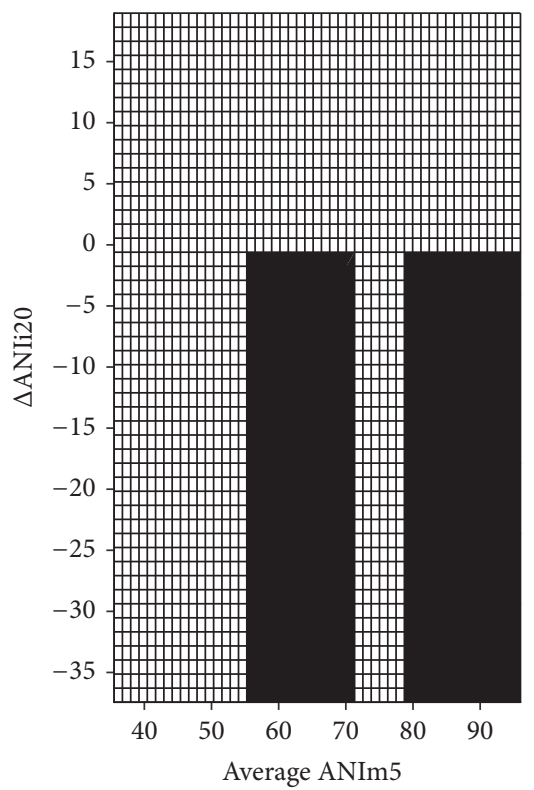

(b)

Figure 9: Comparison of the response surface for the Fuzzy Inference System (a) and for the decision tree (b). Output ranging from 0 (decreasing action) to 100 (increasing action).

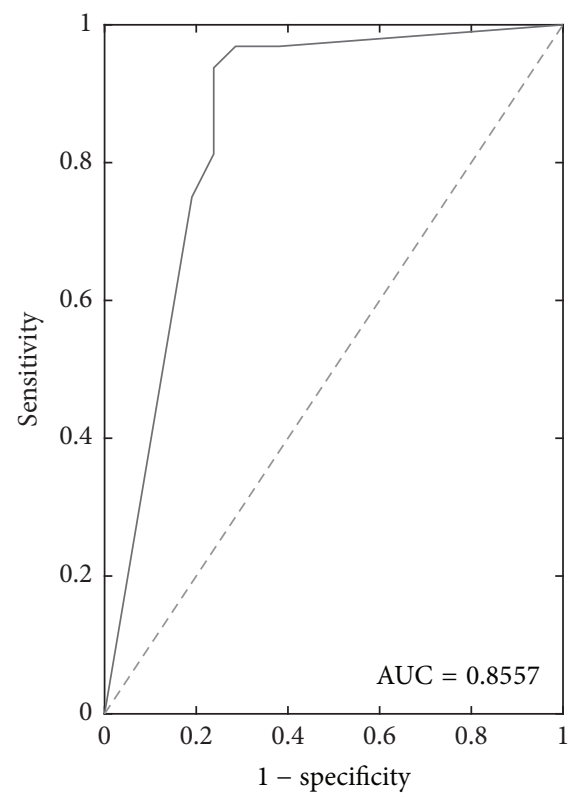

(a)

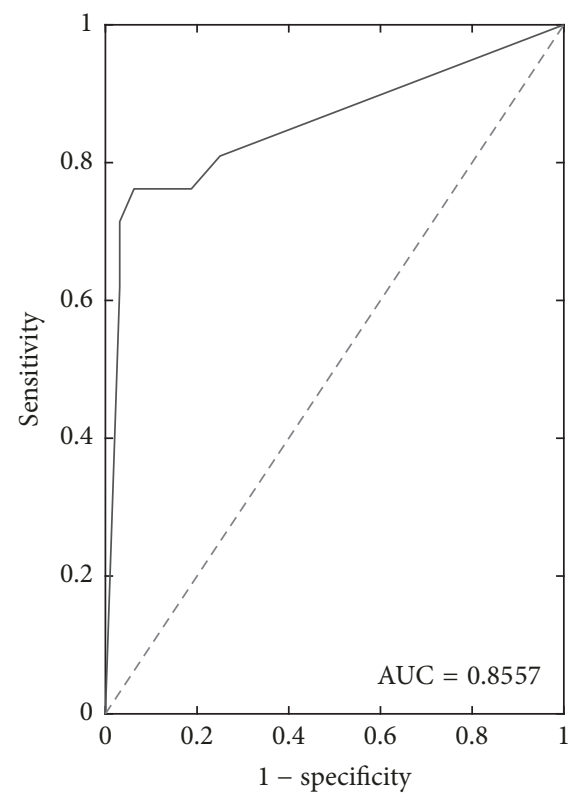

(b)

FIGURE 10: ROC curve showing the relationship between sensitivity (true positive rate) and 1 -specificity (true negative rate) determining the performance of the FIS model proposed to predict the increments (a) and decrements (b) of remifentanil rate. AUC, Area Under the Curve.

it is possible to validate the accuracy of the device to drug assessment as well as to define a new drug delivery protocol. As a result, the knowledge behind the process is automatically built as a set of rules and categories or membership functions. On the one hand, this structure makes it easy to translate the knowledge into an interpretable language for clinicians. What is more, the output expressed as a percentage gives information about the decision and its reliability.
Merging decision trees with fuzzy logic has been previously proposed in order to handle uncertainty, ambiguity, and indeterminacy in the store information. However, unlike our method which results in a Fuzzy Inference System, the previous research has been based on fuzzy decision trees $[76,77]$. They are mainly based on the use of decision tree whose nodes are not crisp values but membership functions. Bockstaller et al. proposed a new fuzzy decision tree for 


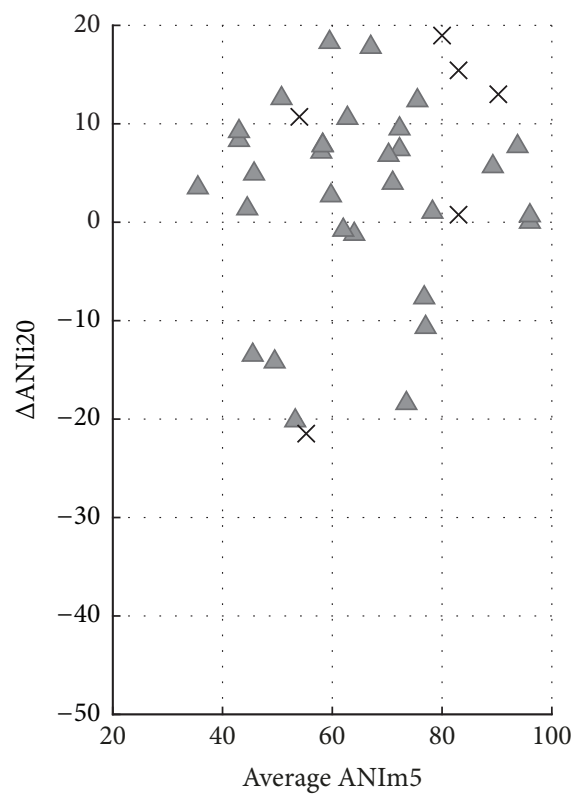

$\triangle$ Correct increment prediction $\times$ Incorrect increment prediction

(a)

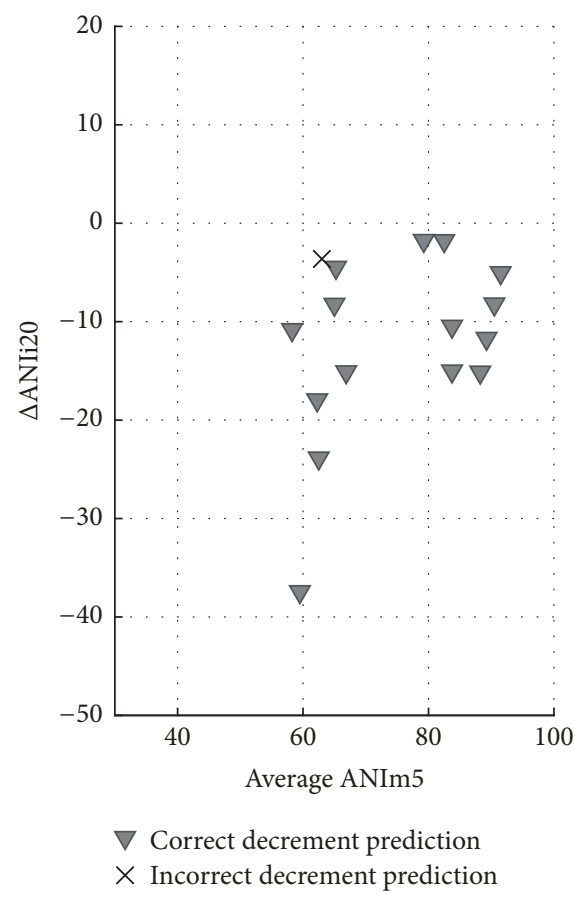

(b)

Figure 11: Prediction of the Fuzzy Inference System for increments (a) and decrements (b) of remifentanil rate.

sustainability assessment [78]. They developed CONTRA tool to support the design of fuzzy decision tree. When using CONTRA, the user has to define the threshold and the limit values of each membership function in a previous step. Moreover, the choice of a weight or rank must be assigned to the different input variables in order to compute the output. Unlike our proposal, a previous heuristic knowledge is necessary in order to design the model. What is more, CONTRA tool limited the input variables to be aggregated between two and five in order to limit a maximum of thirtytwo-decision rules. However, our method was able to work with an unlimited number of inputs.

One of the main limitations to this work is that the presented algorithm has been only applied to a two-decision system (increasing and decreasing drug) in analgesia. Further studies should be considered when applying this algorithm to another similar scenarios such as hypnosis, neuromuscular blockade or glucose control. In addition, including this information to an automatic control system would be the first step in order to automate the analgesic process through a closed-loop strategy.

\section{Conclusion}

This paper introduced a new methodology in order to design a fuzzy-based decision system to improve the drug delivery process when a new guiding variable is involved. Furthermore, the capability of a new monitor to guide the drug titration can be analysed. This methodology involved the whole process: from the recording of numerical data computed by the new monitor to the design of the Fuzzy
Inference System from real data. Fuzzy logic was used as it provides a well-understood mechanism for inducing classification rules from data and avoid possible problems related to the limitation of the number of training data. Rules as well as membership functions were extracted from a decision tree algorithm in order to automate the process. The algorithm proposed was tested in the analgesia scenario. In light of the results, it can be concluded that our method can be used to develop a decision-making system from real data in the medicine field although there exists a lack of knowledge behind the process.

\section{Conflicts of Interest}

The authors declare that there are no conflicts of interest regarding the publication of this article.

\section{Acknowledgments}

This study was funded by the Spanish Ministry of Education, Culture and Sport (http//:www.mecd.gob.es) under the "Formación de Profesorado" Grant FPU15/03347 to Jose M. Gonzalez-Cava.

\section{References}

[1] J.-C. Pomerol, "Artificial intelligence and human decision making," European Journal of Operational Research, vol. 99, no. 1, pp. 3-25, 1997.

[2] J. Lee, J. Ni, D. Djurdjanovic, H. Qiu, and H. Liao, "Intelligent prognostics tools and e-maintenance," Computers in Industry, vol. 57, no. 6, pp. 476-489, 2006. 
[3] P. Holimchayachotikul and K. Leksakul, "Predictive performance measurement system for retail industry using neurofuzzy system based on swarm intelligence," Soft Computing, vol. 21, no. 7, pp. 1895-1912, 2017.

[4] I. Cenamor, T. de la Rosa, S. Núñez, and D. Borrajo, "Planning for tourism routes using social networks," Expert Systems with Applications, vol. 69, pp. 1-9, 2017.

[5] P. Garrido, J. Barrachina, F. J. Martinez, and F. J. Seron, "Smart tourist information points by combining agents, semantics and AI techniques," Computer Science and Information Systems, vol. 14, no. 1, pp. 1-23, 2017.

[6] Z. Wang and R. S. Srinivasan, "A review of artificial intelligence based building energy use prediction: contrasting the capabilities of single and ensemble prediction models," Renewable and Sustainable Energy Reviews, vol. 75, pp. 796-808, 2017.

[7] B. Robson and S. Boray, "Data-mining to build a knowledge representation store for clinical decision support. Studies on curation and validation based on machine performance in multiple choice medical licensing examinations," Computers in Biology and Medicine, vol. 73, pp. 71-93, 2016.

[8] L. Sarangi, M. N. Mohanty, and S. Patnaik, "Design of ANFIS based e-health care system for cardio vascular disease detection," in Recent Developments in Intelligent Systems and Interactive Applications, vol. 541 of Advances in Intelligent Systems and Computing, pp. 445-453, Springer International Publishing, Cham, 2017.

[9] N. Chanamool and T. Naenna, "Fuzzy FMEA application to improve decision-making process in an emergency department," Applied Soft Computing, vol. 43, pp. 441-453, 2016.

[10] L. A. G. Celi, R. J. Tang, M. C. Villarroel, G. A. Davidzon, W. T. Lester, and H. C. Chueh, "A clinical database-driven approach to decision support: predicting mortality among patients with acute kidney injury," Journal of Healthcare Engineering, vol. 2, no. 1, pp. 97-109, 2011.

[11] J. S. de Bruin, K.-P. Adlassnig, A. Blacky, and W. Koller, "Detecting borderline infection in an automated monitoring system for healthcare-associated infection using fuzzy logic," Artificial Intelligence in Medicine, vol. 69, pp. 33-41, 2016.

[12] R. Palaniappan, K. Sundaraj, S. Sundaraj, N. Huliraj, and S. S. Revadi, "A telemedicine tool to detect pulmonary pathology using computerized pulmonary acoustic signal analysis," Applied Soft Computing, vol. 37, pp. 952-959, 2015.

[13] J.-L. Casteleiro-Roca, J. L. Calvo-Rolle, J. A. M. Pérez, N. R. Gutiérrez, and F. J. de Cos Juez, "Hybrid intelligent system to perform fault detection on BIS sensor during surgeries," Sensors, vol. 17, no. 1, article 179, 2017.

[14] D. Nauck and R. Kruse, "Obtaining interpretable fuzzy classification rules from medical data," Artificial Intelligence in Medicine, vol. 16, no. 2, pp. 149-169, 1999.

[15] M. Jakubczyk and B. Kaminski, "Fuzzy approach to decision analysis with multiple criteria and uncertainty in health technology assessment," Annals of Operations Research, vol. 251, no. 1-2, pp. 301-324, 2017.

[16] J. A. Hazelzet, "Can fuzzy logic make things more clear?" Critical Care, vol. 13, no. 1, 2009.

[17] M. J. Gangeh, A. Hashim, A. Giles, L. Sannachi, and G. J. Czarnota, "Computer aided prognosis for cell death categorization and prediction in vivo using quantitative ultrasound and machine learning techniques," Medical Physics, vol. 43, no. 12, pp. 6439-6454, 2016.

[18] Y. Wang, P. Wu, Y. Liu, C. Weng, and D. Zeng, "Learning optimal individualized treatment rules from electronic health record data," in Proceedings of the 2016 IEEE International Conference on Healthcare Informatics, ICHI '16, pp. 65-71, 2016.

[19] M. Dojat, A. Harf, D. Touchard, M. Laforest, F. Lemaire, and L. Brochard, "Evaluation of a knowledge-based system providing ventilatory management and decision for extubation," American Journal of Respiratory and Critical Care Medicine, vol. 153, no. 3, pp. 997-1004, 1996.

[20] H. Ying et al., "A fuzzy discrete event system approach to determining optimal HIV/AIDS treatment regimens," IEEE Transactions on Information Technology in Biomedicine, vol. 10, no. 4, pp. 663-676, 2006.

[21] C. M. Salgado, S. M. Vieira, L. F. Mendonça, S. Finkelstein, and J. M. C. Sousa, "Ensemble fuzzy models in personalized medicine: application to vasopressors administration," Engineering Applications of Artificial Intelligence, vol. 49, pp. 141-148, 2016.

[22] A. S. Fialho, F. Cismondi, S. M. Vieira et al., "Fuzzy modeling to predict administration of vasopressors in intensive care unit patients," in Proceedings of the 2011 IEEE International Conference on Fuzzy Systems, FUZZ '11, pp. 2296-2303, June 2011.

[23] A. Marrero, J. A. Méndez, J. A. Reboso, I. Martín, and J. L. Calvo, "Adaptive fuzzy modeling of the hypnotic process in anesthesia," Journal of Clinical Monitoring and Computing, vol. 31, no. 2, pp. 319-330, 2017.

[24] J. Schäublin, M. Derighetti, P. Feigenwinter, S. Petersen-Felix, and A. M. Zbinden, "Fuzzy logic control of mechanical ventilation during anaesthesia," British Journal of Anaesthesia, vol. 77, no. 5, pp. 636-641, 1996.

[25] D. G. Mason, J. J. Ross, N. D. Edwards, D. A. Linkens, and C. S. Reilly, "Self-learning fuzzy control of atracurium-induced neuromuscular block during surgery," Medical \& Biological Engineering \& Computing, vol. 35, no. 5, pp. 498-503, 1997.

[26] J. A. Méndez, A. Marrero, J. A. Reboso, and A. León, "Adaptive fuzzy predictive controller for anesthesia delivery," Control Engineering Practice, vol. 46, pp. 1-9, 2016.

[27] A. Abad-Gurumeta, J. Ripollés-Melchor, R. Casans-Francés, and J. Calvo-Vecino, "Monitorización de la nocicepción, realidad o ficción?" Revista Española de Anestesiología y Reanimación, vol. 64, no. 7, pp. 406-414, 2017.

[28] M. Gruenewald and C. Ilies, "Monitoring the nociceptionanti-nociception balance," Best Practice \& Research Clinical Anaesthesiology, vol. 27, no. 2, pp. 235-247, 2013.

[29] J.-S. Kang and M.-H. Lee, "Overview of therapeutic drug monitoring," Korean Journal of Internal Medicine, vol. 24, no. 1, pp. 1-10, 2009.

[30] A. S. Gross, "Best practice in therapeutic drug monitoring," British Journal of Clinical Pharmacology, vol. 52, pp. 5-9.

[31] W. V. Tamborlane et al., "Continuous glucose monitoring and intensive treatment of type 1 diabetes," The New England Journal of Medicine, vol. 359, no. 14, pp. 1464-1476, 2008.

[32] B. W. Bode, H. Sabbah, and P. C. Davidson, "What's ahead in glucose monitoring? New techniques hold promise for improved ease and accuracy," Postgraduate Medical Journal, vol. 109, no. 4, pp. 41-49, 2001.

[33] T. Musialowicz and P. Lahtinen, "Current status of EEG-based depth-of-consciousness monitoring during general anesthesia," Current Anesthesiology Reports, vol. 4, no. 3, pp. 251-260, 2014.

[34] M. Hallworth, “Therapeutic drug monitoring," in Clarke's Analysis and Poisons, p. 59, 2011.

[35] M. C. Milone, "Analytical techniques used in therapeutic drug monitoring," Therapeutic Drug Monitoring, pp. 49-73, 2012. 
[36] K. Kuusniemi and R. Pöyhiä, "Present-day challenges and future solutions in postoperative pain management: results from PainForum 2014," Journal of Pain Research, vol. 9, pp. 2536, 2016.

[37] M. M. R. F. Struys, C. Vanpeteghem, M. Huiku, K. Uutela, N. B. K. Blyaert, and E. P. Mortier, "Changes in a surgical stress index in response to standardized pain stimuli during propofolremifentanil infusion," British Journal of Anaesthesia, vol. 99, no. 3, pp. 359-367, 2007.

[38] F. Von Dincklage, H. Velten, B. Rehberg, and J. H. Baars, "Monitoring of the responsiveness to noxious stimuli during sevoflurane mono-anaesthesia by using RIII reflex threshold and bispectral index," British Journal of Anaesthesia, vol. 104, no. 6, pp. 740-745, 2010.

[39] R. K. Ellerkmann, A. Grass, A. Hoeft, and M. Soehle, "The response of the Composite Variability Index to a standardized noxious stimulus during propofol-remifentanil anesthesia," Anesthesia \& Analgesia, vol. 116, no. 3, pp. 580-588, 2013.

[40] B. Hullett, N. Chambers, J. Preuss et al., "Monitoring electrical skin conductance: a tool for the assessment of postoperative pain in children?" Anesthesiology, vol. 111, no. 3, pp. 513-517, 2009.

[41] R. Cowen, M. K. Stasiowska, H. Laycock, and C. Bantel, "Assessing pain objectively: The use of physiological markers," Anaesthesia, vol. 70, no. 7, pp. 828-847, 2015.

[42] R. Logier, M. Jeanne, J. De Jonckheere, A. Dassonneville, M. Delecroix, and B. Tavernier, "PhysioDoloris: a monitoring device for analgesia/nociception balance evaluation using heart rate variability analysis," in Proceedings of the 2010 32nd Annual International Conference of the IEEE Engineering in Medicine and Biology Society, EMBC '10, pp. 1194-1197, September 2010.

[43] A. Gritsan, N. Dovbish, D. Kurnosov, and E. Gritsan, "Control of the adequacy of analgesia during general anesthesia with the use of the monitor analgesia nociception index," Anesthesia \& Analgesia, vol. 123, 2016.

[44] E. Boselli, M. Daniela-Ionescu, G. Bégou et al., "Prospective observational study of the non-invasive assessment of immediate postoperative pain using the analgesia/nociception index (ANI)," British Journal of Anaesthesia, vol. 111, no. 3, pp. 453-459, 2013.

[45] M. Gruenewald, T. Schoenherr, J. Herz, C. Ilies, A. Fudickar, and B. Bein, "Analgesia Nociception Index (ANI) for detection of noxious stimulation during sevofluran-remifentanil anaesthesia," European Journal of Anaesthesiology, vol. 30, pp. 223-223, 2013.

[46] L. Ursulet, J. Cros, J. De Jonckheere, P. Senges, A. Vincelot, and N. Nathan, "Bedside analysis of heart rate variability by Analgesia Nociception Index (ANI) predicts hypotension after spinal anesthesia for elective Caesarean delivery," European Journal of Anaesthesiology, vol. 29, p. 5, 2012.

[47] M. Jeanne, C. Clément, J. De Jonckheere, R. Logier, and B. Tavernier, "Variations of the analgesia nociception index during general anaesthesia for laparoscopic abdominal surgery," Journal of Clinical Monitoring and Computing, vol. 26, no. 4, pp. 289-294, 2012.

[48] M. Sesay, A. Mainchain, M. Biais, D. Liguoro, and K. NouetteGaulain, "Impact of the Anesthesia Nociception Index on remifentanil consumption during anterior cervical discectomy," Anesthesia and Analgesia, vol. 123, p. 428, 2016.

[49] G. Daccache, S. Goursaud, E. Lemasson, L. Berger, J. Fellahi, and J. Hanouz, "Target-controlled dosing of remifentanil guided by the analgesia nociception index: a feasibility study," European Journal of Anaesthesiology, vol. 31, p. 35, 2014.

[50] M. Gruenewald, C. Ilies, J. Herz et al., "Influence of nociceptive stimulation on analgesia nociception index (ANI) during propofol-remifentanil anaesthesia," British Journal of Anaesthesia, vol. 110, no. 6, pp. 1024-1030, 2013.

[51] G. Jess, E. M. Pogatzki-Zahn, P. K. Zahn, and C. H. MeyerFrieem, "Monitoring heart rate variability to assess experimentally induced pain using the analgesia nociception index," European Journal of Anaesthesiology, vol. 33, no. 2, pp. 118-125, 2016.

[52] Q. Yan, H. Y. An, and Y. Feng, "Pain assessment in conscious healthy volunteers: a crossover study evaluating the analgesia/nociception index," British Journal of Anaesthesia, vol. 118, no. 4, pp. 635-636, 2017.

[53] P. S. Myles and N. Urquhart, "The linearity of the visual analogue scale in patients with severe acute pain," Anaesthesia and Intensive Care Journal, vol. 33, no. 1, pp. 54-58, 2005.

[54] C. A. Bodian, G. Freedman, S. Hossain, J. B. Eisenkraft, and Y. Beilin, "The visual analog scale for pain: clinical significance in postoperative patients," Anesthesiology, vol. 95, no. 6, pp. 13561361, 2001.

[55] J. A. Szental, A. Webb, C. Weeraratne, A. Campbell, H. Sivakumar, and S. Leong, "Postoperative pain after laparoscopic cholecystectomy is not reduced by intraoperative analgesia guided by analgesia nociception index $\left(\mathrm{ANI}^{\circledR}\right)$ monitoring: a randomized clinical trial," British Journal of Anaesthesia, vol. 114, no. 4, pp. 640-645, 2015.

[56] A. Castro, F. G. de Almeida, P. Amorim, and C. S. Nunes, "A novel multivariate STeady-state index during general ANesthesia (STAN)," Journal of Clinical Monitoring and Computing, vol. 31, no. 4, pp. 851-860, 2017.

[57] M. Jeanne, M. Delecroix, J. De Jonckheere, A. Keribedj, R. Logier, and B. Tavernier, "Variations of the analgesia nociception index during propofol anesthesia for total knee replacement," The Clinical Journal of Pain, vol. 30, no. 12, pp. 1084-1088, 2014.

[58] E. Boselli, R. Logier, L. Bouvet, and B. Allaouchiche, "Prediction of hemodynamic reactivity using dynamic variations of Analgesia/Nociception Index (Delta ANI)," Journal of Clinical Monitoring and Computing, vol. 30, no. 6, pp. 977-984, 2016.

[59] V. Podgorelec, P. Kokol, B. Stiglic, and I. Rozman, "Decision trees: an overview and their use in medicine," Journal of Medical Systems, vol. 26, no. 5, pp. 445-463, 2002.

[60] T. G. Dietterich, "Experimental comparison of three methods for constructing ensembles of decision trees: bagging, boosting, and randomization," Machine Learning, vol. 40, no. 2, pp. 139157,2000

[61] A. Colin, "Building decision trees with the ID3 algorithm," Dr. Dobb's Journal, vol. 21, no. 6, pp. 107-109, 1996.

[62] L. Breiman, J. H. Friedman, R. A. Olshen, and C. J. Stone, Classification and Regression Trees, vol. 19, 1984.

[63] A. Venkatasubramaniam, J. Wolfson, N. Mitchell, T. Barnes, M. Jaka, and S. French, "Decision trees in epidemiological research," Emerging Themes in Epidemiology, vol. 14, no. 1, article 11, 2017.

[64] X. Wu and V. Kumar, "The Top Ten Algorithms in Data Mining," 2009.

[65] R. Kohavi, "A study of cross-validation and bootstrap for accuracy estimation and model selection," in Proceedings of the 14th International Joint Conference on Artificial Intelligence, vol. 2, pp. 1137-1143, 1995. 
[66] P.-N. Tan, M. Steinbach, and V. Kumar, Introduction to Data Mining, Addison-Wesley Longman Publishing Co., Inc., Massachusetts, Mass, USA, 5th edition, 2005.

[67] H. Kehlet and K. Holte, "Effect of postoperative analgesia on surgical outcome," British Journal of Anaesthesia, vol. 87, no. 1, pp. 62-72, 2001.

[68] H. Kehlet, T. S. Jensen, and C. J. Woolf, "Persistent postsurgical pain: risk factors and prevention," The Lancet, vol. 367, no. 9522, pp. 1618-1625, 2006.

[69] P. Gueth, D. Dauvergne, N. Freud et al., "Machine learningbased patient specific prompt-gamma dose monitoring in proton therapy," Physics in Medicine and Biology, vol. 58, no. 13, pp. 4563-4577, 2013.

[70] C. Huang, R. Mezencev, J. F. McDonald, F. Vannberg, and B. Liu, "Open source machine-learning algorithms for the prediction of optimal cancer drug therapies," PLoS ONE, vol. 12, no. 10, 2017.

[71] M. Gram, J. Erlenwein, F. Petzke et al., "Prediction of postoperative opioid analgesia using clinical-experimental parameters and electroencephalography," European Journal of Pain, vol. 21, no. 2, pp. 264-277, 2017.

[72] H. F. Galley, "II. Solid as a ROC," British Journal of Anaesthesia, vol. 93, no. 5, pp. 623-626, 2004.

[73] N. R. Pal and S. Chakraborty, "Fuzzy rule extraction from ID3type decision trees for real data," IEEE Transactions on Systems, Man, and Cybernetics, Part B: Cybernetics, vol. 31, no. 5, pp. 745754, 2001.

[74] K. Nozaki, H. Ishibuchi, and H. Tanaka, "Adaptive fuzzy rule-based classification systems," IEEE Transactions on Fuzzy Systems, vol. 4, no. 3, pp. 238-250, 1996.

[75] J. S. R. Jang, "ANFIS: adaptive-network-based fuzzy inference system," IEEE Transactions on Systems, Man, and Cybernetics, vol. 23, no. 3, pp. 665-685, 1993.

[76] C. Olaru and L. Wehenkel, "A complete fuzzy decision tree technique," Fuzzy Sets and Systems, vol. 138, no. 2, pp. 221-254, 2003.

[77] X. Wang, X. Liu, W. Pedrycz, and L. Zhang, "Fuzzy rule based decision trees," Pattern Recognition, vol. 48, no. 1, pp. 50-59, 2015.

[78] C. Bockstaller, S. Beauchet, V. Manneville, B. Amiaud, and R. Botreau, "A tool to design fuzzy decision trees for sustainability assessment," Environmental Modeling and Software, vol. 97, pp. 130-144, 2017. 


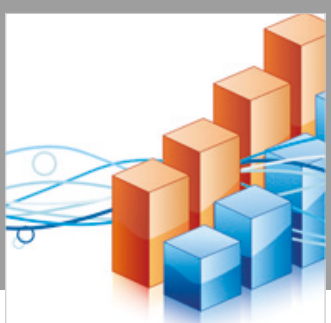

Advances in

Operations Research

\section{-n-m}
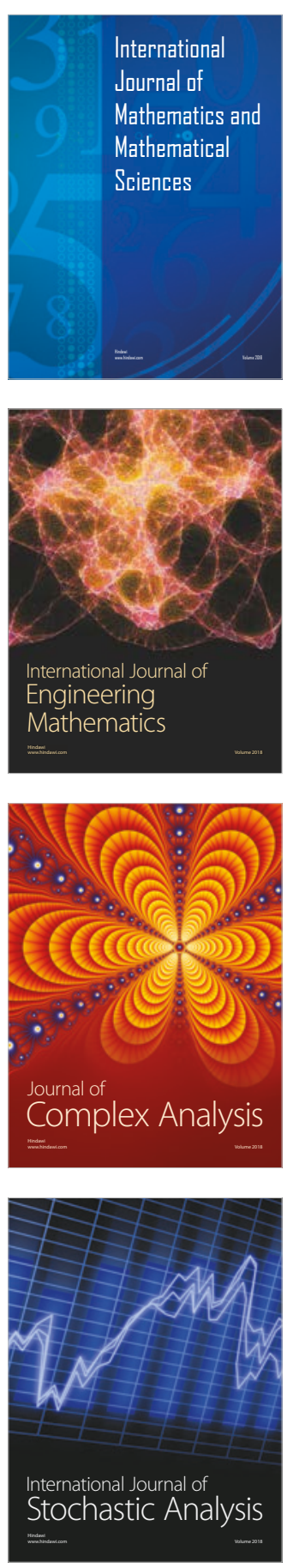
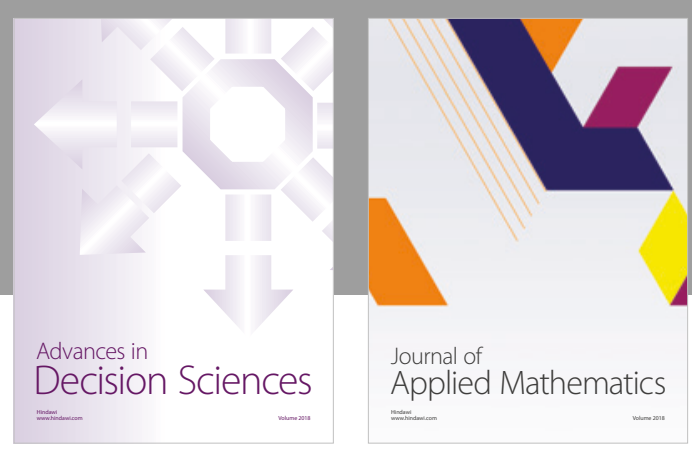

Journal of

Applied Mathematics
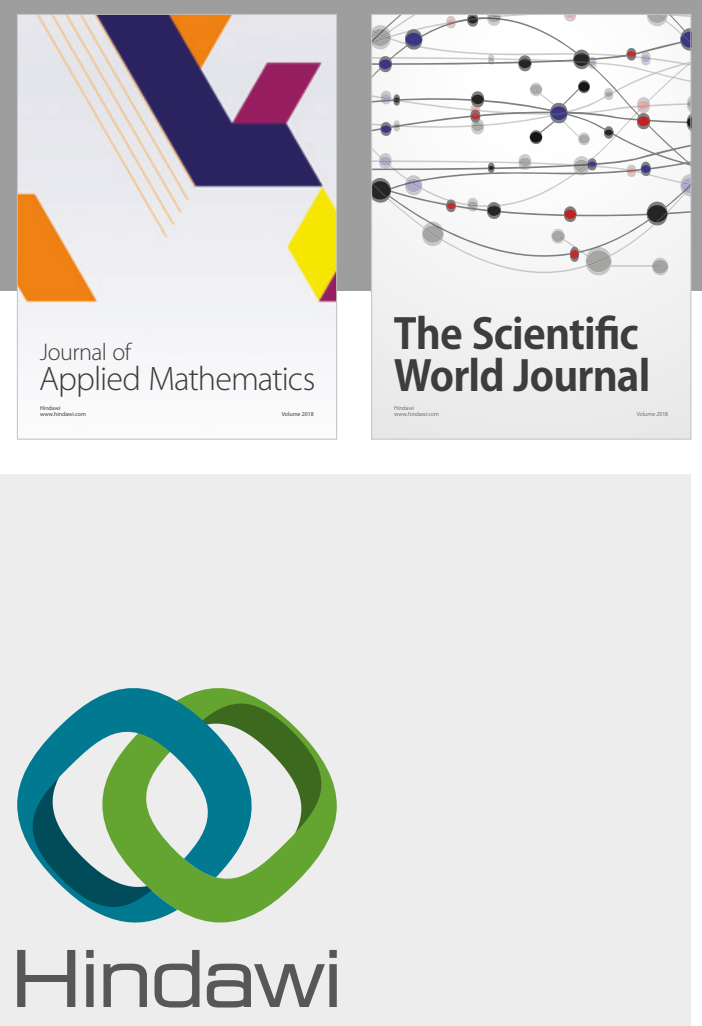

Submit your manuscripts at

www.hindawi.com

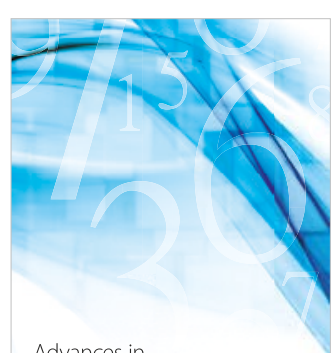

Advances in
Numerical Analysis
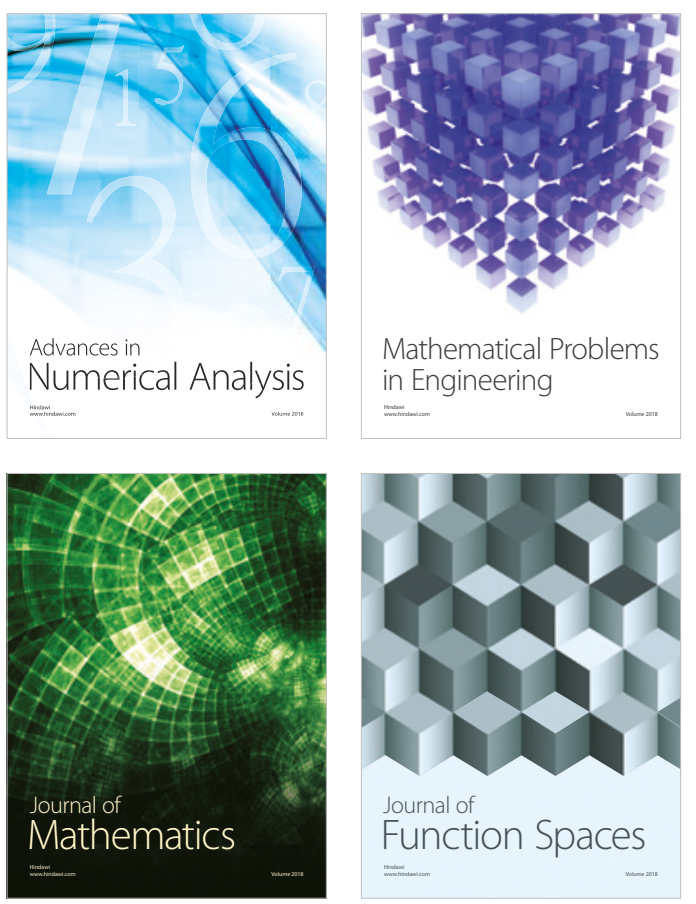

Mathematical Problems in Engineering

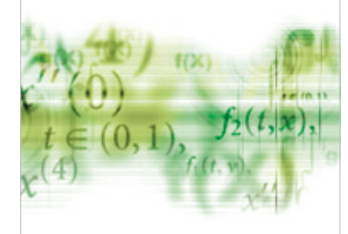

International Journal of

Differential Equations

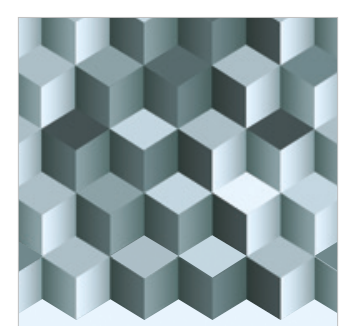

Journal of

Function Spaces
The Scientific

World Journal

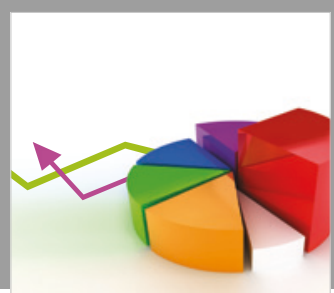

Journal of

Probability and Statistics
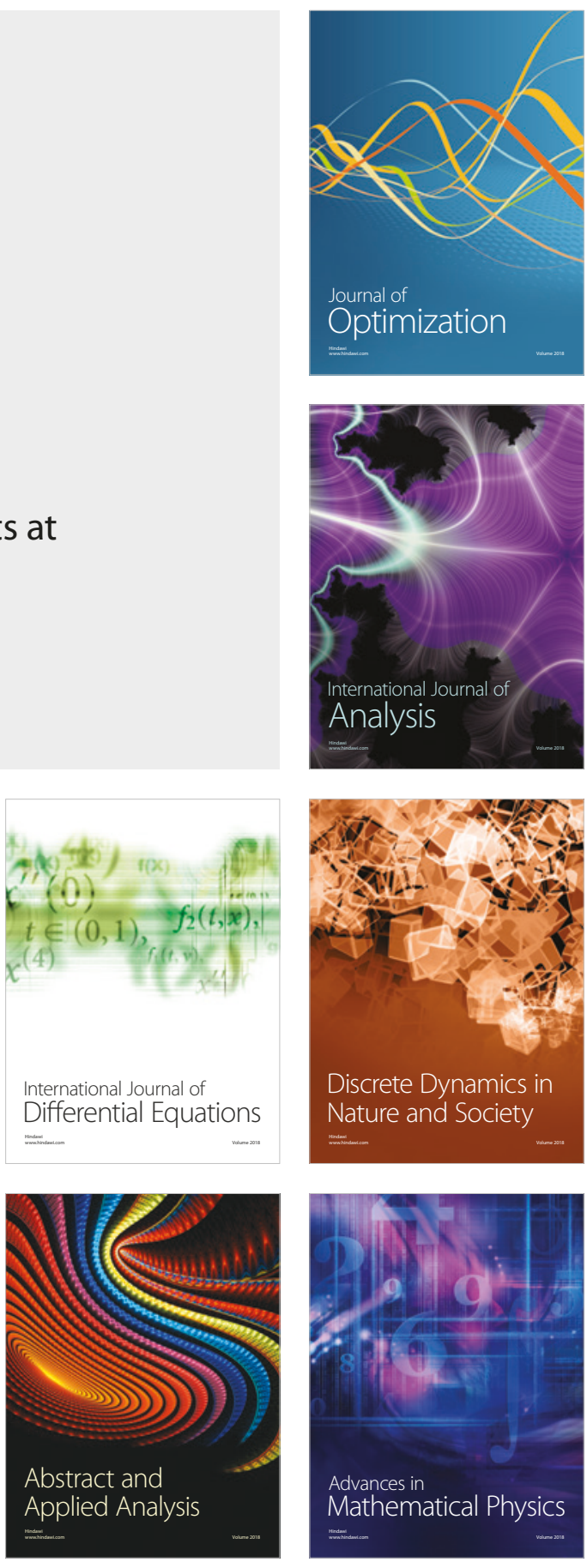